\title{
EFEITO DE EXPERIMENTOS EM AULAS PRÁTICAS DE METABOLISMO COM O CORPO DOS PRÓPRIOS ESTUDANTES UNIVERSITÁRIOS SOBRE O SEU NÍVEL DE APRENDIZAGEM
}




\title{
EFEITO DE EXPERIMENTOS EM AULAS PRÁTICAS DE METABOLISMO COM O CORPO DOS PRÓPRIOS ESTUDANTES UNIVERSITÁRIOS SOBRE O SEU NÍVEL DE APRENDIZAGEM
}

\author{
Dissertação apresentada ao Programa de \\ Pós-graduação "Stricto Sensu” em Ciências \\ e Tecnologias em Saúde da Universidade \\ de Brasília, como requisito para a obtenção \\ do título de Mestre em Ciências e \\ Tecnologias em Saúde.
}

Orientador: Prof. Dr. Alexis Fonseca Welker

Brasília 
Ficha catalográfica elaborada pela Biblioteca Central da Universidade de Brasília. Acervo 1020843.

Eduardo, Anna Maly de Leăo e Neves.

E24e Efeito de experimentos em aulas práticas de metabol ismo cam o corpo dos próprios estudantes universitários sobre o seu nível de aprendizagem / Anna $\mathrm{Maly}$ de Leão e Neves Eduardo. -. 2015.

viii, $38 \mathrm{f} .:$ il. ; $30 \mathrm{~cm}$.

Dissertação (mestrado) - Universidade de Brasília, Programa de Pós-Graduação em Ciências e Tecnologias em Saúde, 2015.

Orientação: Alexis Fonseca Welker.

Inclui bibliografia.

1. Saúde - Estudo e ensino (Superior). 2. Estudantes universitários - Avaliação. 3. Aprendi zagem. 4. Mbti vaçăo na educaçăo. I. Welker, Alexis Fonseca. II. Título. 



\section{ANNA MALY DE LEÃO E NEVES EDUARDO}

\section{EFEITO DE EXPERIMENTOS EM AULAS PRÁTICAS DE METABOLISMO COM O CORPO DOS PRÓPRIOS ESTUDANTES UNIVERSITÁRIOS SOBRE O SEU NÍVEL DE APRENDIZAGEM}

Dissertação defendida no Programa de Pós-graduação "Stricto Sensu" em Ciências e Tecnologias em Saúde da Universidade de Brasília, como parte das exigências para a obtenção do título de Mestre em Ciências e Tecnologias em Saúde defendida em 06 de março de 2015 para a banca examinadora constituída pelos seguintes membros:

Prof. Dr. Alexis Fonseca Welker

Universidade de Brasília

Programa de Pós-graduação em Ciências e Tecnologias em Saúde da Universidade de Brasília

Prof. Dr. Wagner Fontes

Universidade de Brasília

Programa de Pós-graduação em Ensino de Ciências

Profa. Dra. Tatiana Ramos Lavich

Universidade de Brasília

Faculdade de Ceilândia

Prof. Dr. Fernando Fortes de Valência (suplente)

Universidade de Brasília

Departamento de Biologia Celular 


\section{SUMÁRIO}

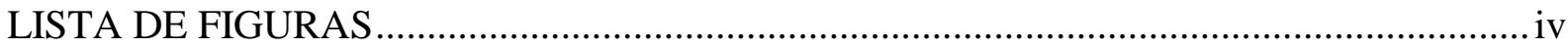

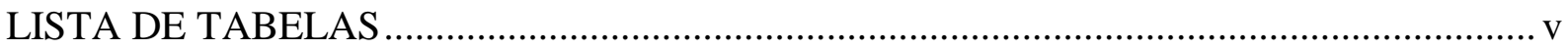

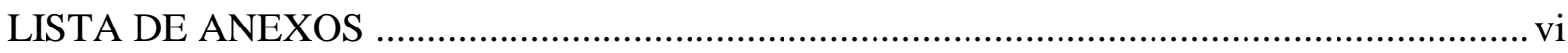

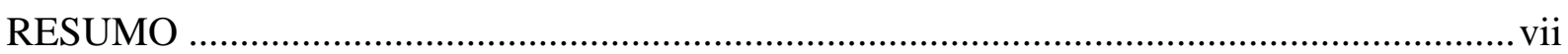

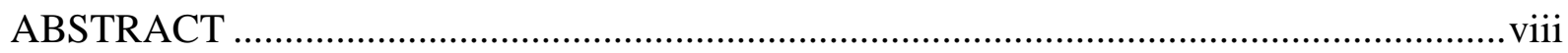

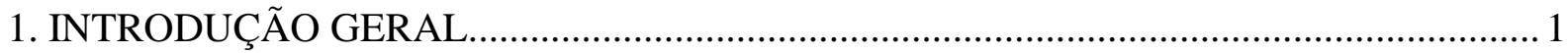

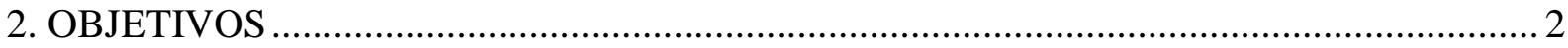

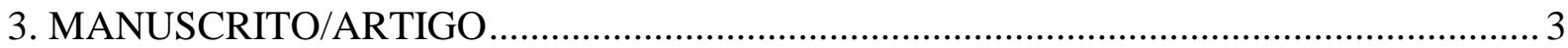

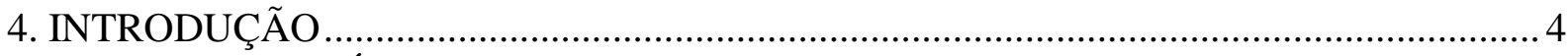

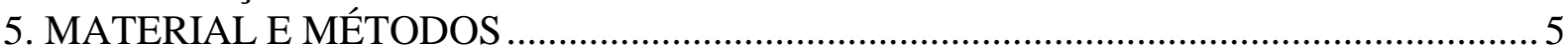

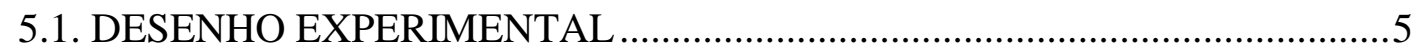

5.2. SUJEITOS E DESCRIÇÃO DO CURSO.....................................................

5.3. PROCEDIMENTOS DOS GRUPOS CONTROLE E TRATAMENTO...........6

5.4. AVALIAÇÃO DO DESEMPENHO E DA MOTIVAÇÃO ............................

5.5. DESCRIÇÃO E ANÁLISE ESTATÍSTICA …................................................

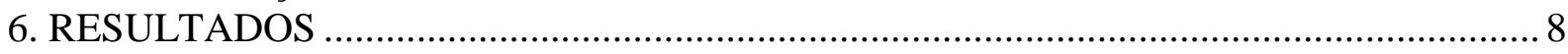

6.1. INDICADORES DE APRENDIZAGEM......................................................

6.2. PERCEPÇÃO SOBRE AS AULAS PRÁTICAS............................................ 10

6.3. COMPARAÇÃO DA ESCALA DE MOTIVAÇÃO ....................................12

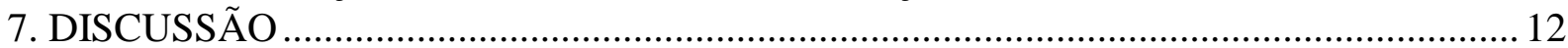

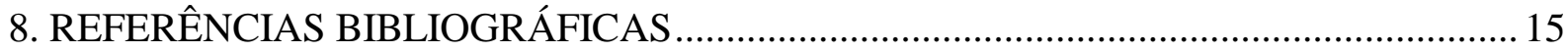

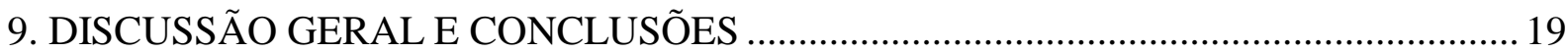

10. RECOMENDAÇÕES PARA ESTUDOS FUTUROS E CONCLUSÃO ......................... 21

11. REFERÊNCIAS BIBLIOGRÁFICAS (das partes não pertencentes ao manuscrito) ........... 23

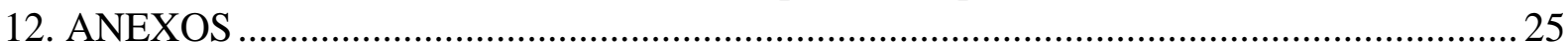




\section{LISTA DE FIGURAS}

FIGURA 1. EFEITO DO EXPERIMENTO USANDO O PRÓPRIO ORGANISMO DE ESTUDANTES QUE SOMENTE OBSERVARAM OU QUE DOARAM SANGUE SOBRE O SEU RENDIMENTO EM AVALIAÇÕES DE APRENDIZAGEM. *: $\mathrm{P}<0,05$. \#: $\mathrm{P}<0,05.9$

FIGURA 2. EFEITO DO EXPERIMENTO USANDO O PRÓPRIO ORGANISMO DE TODOS OS ESTUDANTES QUE PARTICIPARAM ATIVAMENTE DOANDO SANGUE E QUE PERMANECERAM EM JEJUM OU INGERIRAM GLICOSE OU FRUTOSE. *: P < 0,05 .

FIGURA 3. GRAU DE MOTIVAÇÃO DOS ESTUDANTES QUE PARTICIPARAM ATIVAMENTE DOANDO SANGUE. 12 


\section{LISTA DE TABELAS}

TABELA 1. CONTEÚDOS DA DISCIPLINA DE BIOLOGIA CELULAR. 6

TABELA 2. MEDIDAS DESCRITIVAS PARA AS PROVAS A1 E A2 ENTRE OS OBSERVADORES E ATIVOS

TABELA 3. FREQUÊNCIA E MEDIDAS DESCRITIVAS PARA AS VARIÁVEIS DOS ATIVOS

TABELA 4. MEDIDAS DESCRITIVAS PARA AS QUESTÕES (Q1-Q5) ENTRE OS ATIVOS E OBSERVADORES. 


\section{LISTA DE ANEXOS}

ANEXO A - APROVAÇÃO DO COMITÊ DE ÉTICA EM PESQUISA ................................ 25

ANEXO B - TERMO DE CONSENTIMENTO LIVRE E ESCLARECIDO (TCLE) ............ 28

ANEXO C - TERMO DE RESPONSABILIDADE E COMPROMISSO DA

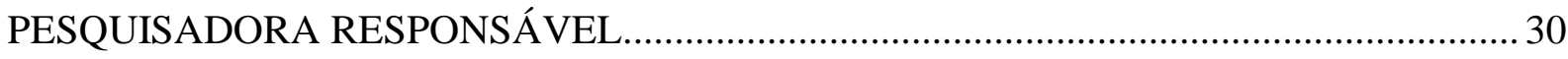

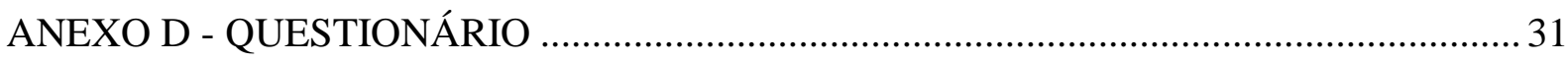

ANEXO E - CLASSIFICAÇÃO QUALIS CAPES DA REVISTA “ADVANCES IN PHYSIOLOGY EDUCATION”.

ANEXO F - INSTRUÇÕES A AUTORES PARA SUBMISSÃO DE MANUSCRITO À REVISTA “ADVANCES IN PHYSIOLOGY EDUCATION” 


\section{RESUMO}

Eduardo, Anna Maly De Leão e Neves. Efeito de experimentos em aulas práticas de metabolismo com o corpo dos próprios estudantes universitários sobre o seu nível de aprendizagem. 2015. Dissertação (Mestrado em Ciências e Tecnologias em Saúde da Universidade de Brasília) - Programa de Pós-graduação "Stricto Sensu” em Ciências e Tecnologias em Saúde da Universidade de Brasília, UnB.

Vários estudos mostram que metodologias de ensino de aprendizagem ativa são mais atrativas e geram maior interação do que metodologias mais passivas, sendo que aquelas que simulam situações cotidianas tendem a promover maior aprendizado. Outra estratégia que pode aumentar o rendimento acadêmico é o uso de métodos de auto-monitoramento e autoavaliação. Até onde sabemos, não existem estudos que avaliaram o impacto de aulas práticas usando o próprio corpo em situações rotineiras sobre o aprendizado, ou seja, que envolvam as duas estratégias, tanto atividades do cotidiano quanto auto-monitoramento. Este estudo objetivou analisar o impacto de experimentos usando o próprio organismo em condições que mimetizam situações observadas no cotidiano - jejum e ingestão de refeições ricas em carboidratos - sobre o rendimento nas avaliações de estudantes universitários da área da saúde e no grau de motivação em realizar estes experimentos. A metodologia de ensino utilizada neste estudo aumentou as notas dos estudantes em provas de conhecimento, indicando um aumento do aprendizado, quando comparados ao grupo controle, que apenas observou as experiências. Além disso, a análise dos três subgrupos que participaram ativamente do experimento mostrou que aqueles que ingeriram soluções de glicose e frutose tiveram aumento significativo de nota, enquanto que o que permaneceu em jejum não. Tal diferença indica que a metodologia associando duas estratégias diferentes, a aprendizagem ativa e a auto avaliação, aumenta o grau de atenção às aulas. O instrumento de ensino apresentado no presente trabalho mostrou ser uma alternativa positiva para estudantes universitários da área da saúde.

Palavras-chave: Experimentos no próprio corpo. Metodologia de aprendizagem ativa. Motivação em estudar. Aula prática. 


\begin{abstract}
Eduardo, Anna Maly De Leão e Neves. Effect of experiments in practical classes on metabolism with the own university student's body on their level of learning. 2015. Dissertação (Mestrado em Ciências e Tecnologias em Saúde da Universidade de Brasília) Programa de Pós-graduação "Stricto Sensu” em Ciências e Tecnologias em Saúde da Universidade de Brasília, UnB.
\end{abstract}

Several studies show that methodologies of active learning are more attractive and generate more interaction than more passive methods, and those that simulate everyday situations tend to promote higher learning. Another strategy that can increase academic performance is the use of self-monitoring and self-evaluation methods. To our knowledge, there are no studies evaluating the impact of practical classes using the student's body in routine situations on learning, involving the two strategies, both daily activities and selfmonitoring. This study aimed to analyze the impact of using the body's own experiments in conditions that mimic situations encountered in daily life - fasting and eating meals rich in carbohydrates - in the evaluations of university students in the health field and the degree of motivation to perform these experiments. The teaching methodology used in this study increased the scores of students in tests of knowledge, indicating an increase in learning, when compared to the control group, which only observed the experiences. Furthermore, analysis of the three subgroups that actively participate in the experiment showed that those who ingested glucose and fructose solutions have significant increase the scores, while the unfed not. This difference indicates that the methodology involving two different strategies, active learning and self assessment, increases the degree of attention in class. The teaching tool presented in this paper showed a positive alternative for college students in the health field.

Key words: Experiments in the own body. Methodology of active learning. Motivation to study. Practical class. 


\section{INTRODUÇÃO GERAL}

Recentes propostas educacionais publicadas têm mostrado uma preocupação clara e global no que diz respeito ao ensino das áreas básicas da saúde. Com grande conteúdo para cobrir e uma disponibilidade limitada de tempo (Boyer, 2000), o professor tem que ser criativo na seleção dos temas básicos para as palestras e seminários (Hermes-Lima et al., 2002). O contexto atual de mudanças e desafios na formação de profissionais da saúde exige alterações em diferentes esferas - conceituais, interpessoais, sociais, institucionais e de valores, para que a formação desses profissionais ocorra de forma sólida e crítica, além de incluir a construção de alternativas ainda desconhecidas (Feuerwerker, 2002).

Como os alunos estão em relação à sua aprendizagem tem sido um dos interesses fundamentais de pesquisadores educacionais. Esse tópico foi investigado em muitos estágios da educação formal (Rozendaal et al., 2003; Van Bragt et al., 2007), e um dos mais importantes a serem estudados no ensino superior. A aprendizagem é um processo ativo no qual o aluno e o professor têm que trabalhar mutuamente para tornar o processo de compartilhamento de conhecimento agradável e mais fácil para a compreensão (Saville et al., 2006).

Mais recentemente, no entanto, tem havido uma discussão adicional dos efeitos diretos de testes sobre a aprendizagem dos alunos (Larsen et al., 2008). Muitos estudos relatam que através das metodologias ativas de aprendizagem, os alunos podem apresentar melhores notas em provas quando comparados a alunos que apenas têm contato com o sistema mais tradicional de ensino (Erwin e Rieppi, 1999; Cruz e Carvalho, 2007; Narula et al., 2012). Por exemplo, a observação concreta de uma reação química pode motivar e facilitar a compreensão dos alunos sobre a química da vida (Figueira e Rocha, 2013). As metodologias ativas de aprendizagem costumam com frequência usar a problematização como estratégia de aprendizagem, com o intuito de motivar o estudante. A aplicação deste instrumento de ensino se mostra uma metodologia aceitável para os alunos, sendo uma maneira mais interativa comparada às aulas tradicionais em sala (Ghosh et al., 2000; Everly, 2013). Porém, os métodos de ensino baseados em aprendizagem ativa podem não proporcionar um desempenho melhor dos estudantes (Barker, 1988; Persky et al., 2011; Rivkin et al., 2013). 
Experimentos realizados em aulas práticas, onde os estudantes têm a possibilidade de utilizar o próprio corpo e aprender através dele, podem ser um instrumento de ensino interessante e contribuir para uma maior aprendizagem dos alunos. Frente às diversas possibilidades e aos resultados satisfatórios apresentados, quando se aplica um instrumento de ensino baseado na metodologia de aprendizagem ativa, este trabalho torna-se justificável, e a motivação para realizá-lo é bastante pertinente. $\mathrm{O}$ presente trabalho investigou uma metodologia de ensino ativa, com uma aula prática na qual o organismo de estudantes universitários e as suas reações foram testadas frente a soluções de diferentes carboidratos e ao jejum. As notas das provas realizadas antes e depois dos experimentos foram comparadas, bem como a motivação e a satisfação dos estudantes universitários do curso de farmácia em participar de maneira ativa ou como observadores de tal experiência prática.

\section{OBJETIVOS}

O objetivo geral do trabalho foi analisar o impacto de experimentos usando o próprio organismo em condições que mimetizam situações observadas no cotidiano jejum, ingestão de refeição rica em diferentes carboidratos - sobre o rendimento nas avaliações e no grau de motivação em realizar estes experimentos.

Os objetivos específicos foram:

1. Avaliar o grau de motivação e satisfação em realizar experimentos investigando a resposta do próprio organismo ou dos colegas de classe ao jejum e à ingestão de diferentes alimentos.

2. Comparar as notas das provas feitas antes e depois da aplicação da metodologia a ser testada dos diferentes grupos de estudantes. 


\section{MANUSCRITO/ARTIGO}

Os principais resultados e conhecimentos produzidos da presente dissertação estão dispostos no manuscrito/artigo abaixo a ser submetido para publicação em revista.

\section{Efeito de experimentos em aulas práticas de metabolismo com o corpo dos próprios estudantes universitários sobre o seu nível de aprendizagem}

Anna Maly de Leão e Neves Eduardo ${ }^{1,2}$ e Alexis F. Welker, Dr. ${ }^{2^{*}}$

${ }^{1 .}$ Faculdade Anhanguera de Brasília, Curso de Farmácia, Brasília, Brasil.

${ }^{2}$ Universidade de Brasília, UNB - Campus Ceilândia, Curso de Fisioterapia. Brasília, Brasil.

*Corresponding author:

Dr. Alexis Fonseca Welker

Universidade de Brasília, UNB - Campus Ceilândia, QNN 14 Área Especial, Ceilândia, DF CEP 72220-140

Phone: +55-61-3107-8933

Email: welker.af@gmail.com 
Palavras-chave: Experimentos no próprio corpo. Metodologia de aprendizagem ativa. Motivação em estudar. Aula prática.

\section{INTRODUÇÃO}

Vários estudos mostram que metodologias de ensino de aprendizagem ativa são mais atrativas e geram maior interação do que metodologias mais passivas $(14,13,16)$. Dentre as estratégias de aprendizagem ativa, alguns trabalhos indicam que práticas educacionais que mimetizem atividades rotineiras aumentam o aprendizado em relação às práticas sem tal característica. Por exemplo, o uso de pacientes virtuais por estudantes de medicina pode aumentar o grau de aprendizagem (11) e o uso de aprendizagem baseada em casos aumenta a aprendizagem profunda, o envolvimento dos alunos e interesse pelos conteúdos $(20,18)$. Outro método que frequentemente envolve simulações de situações reais (ex. diagnóstico de um paciente hipotético com alguns sintomas), aprendizagem baseada em problemas (PBL), tem se mostrado uma ferramenta importante para enriquecer a retenção de conhecimentos $(25,27,31)$.

Outra estratégia de aprendizagem ativa que pode aumentar o rendimento acadêmico é o uso de métodos de auto-monitoramento e auto-avaliação $(28,34,21,10)$. Por exemplo, após responder perguntas de um teste de conhecimentos, quando os estudantes são permitidos a ver as respostas dos colegas e revisar suas respostas, o número de acertos após a revisão aumenta (1).

Há indicações de que práticas que envolvam ambos, tanto atividades do cotidiano quanto auto-monitoramento, propiciem uma ainda maior atração dos alunos pelas aulas $(19,17)$, bem como maior interação durante as aulas (13). Este é o caso de um estudo que submeteu estudantes universitários à ingestão de pizza e massa e à medição de parâmetros bioquímicos, o que tornou possível cobrir no exame final temas importantes do metabolismo e demonstrar como a máquina metabólica do organismo reage às dietas diferentes. No período em que tal metodologia foi aplicada, as notas foram maiores do que nos períodos anteriores. Porém, não houve grupo controle, impedindo o real conhecimento da eficiência desse método (26). Outros estudos nos quais os estudantes utilizaram o próprio corpo não investigaram a influência da prática 
sobre o aprendizado (22). Os poucos relatos de práticas usando o próprio corpo frequentemente envolveram atividades que não fazem parte da rotina dos estudantes (8).

Até onde sabemos, não existem estudos que avaliaram o impacto de aulas práticas usando o próprio corpo em situações rotineiras sobre o aprendizado. Um experimento que envolvesse a mensuração do rendimento acadêmico de estudantes que têm aulas práticas usando o próprio corpo, comparado a aulas práticas comuns (controle), poderia mostrar a influência de tais aulas sobre o aprendizado dos alunos. Este trabalho pode apresentar uma nova metodologia ativa mais eficiente do que as metodologias comumente empregadas nas salas de aula.

Este estudo objetivou analisar o impacto de experimentos usando o próprio organismo em condições que mimetizam situações observadas no cotidiano - jejum e ingestão de refeições ricas em carboidratos - sobre o rendimento nas avaliações de estudantes universitários da área da saúde e no grau de motivação e satisfação em realizar estes experimentos.

\section{MATERIAL E MÉTODOS}

\subsection{DESENHO EXPERIMENTAL}

Para analisar o impacto de realizar experimentos usando o próprio organismo, sobre o aprendizado do conhecimento da área da saúde, parte de uma turma de estudantes universitários do curso de farmácia participou ativamente de um experimento, enquanto que a outra parte, apenas acompanhou o estudo. $\mathrm{O}$ experimento permitia aos estudantes estudarem alguns dos conteúdos abordados na disciplina Biologia Celular. Ele envolveu coleta de amostras de sangue do próprio corpo em situações que mimetizam algumas observadas no cotidiano - jejum e ingestão de diferentes carboidratos. Além disso, foi avaliado o efeito do experimento sobre o grau de motivação e satisfação dos estudantes. Os dados desta dissertação foram coletados ao longo do ano de 2013, por uma única pesquisadora.

\subsection{SUJEITOS E DESCRIÇÃO DO CURSO}


Este estudo foi realizado com 71 estudantes do segundo semestre do curso de Farmácia, durante a disciplina de Biologia Celular, na Faculdade Anhanguera de Brasília e $33(46,5 \%)$ participaram ativamente da pesquisa enquanto que o restante apenas observou experimentos feitos pelos primeiros. Nesta disciplina, os estudantes estudam os tipos celulares, os componentes celulares e as suas respectivas funções, durante um semestre, com carga horária total de 60 horas. A Tabela 1 mostra os conteúdos da disciplina. A avaliação do nível de conhecimento dos estudantes ocorreu através de duas provas escritas individuais e sem consulta e da aplicação de questionários sobre os temas estudados em sala de aula. As avaliações foram iguais para todos os grupos. Este trabalho teve seu projeto aprovado por Comitê de Ética e Pesquisa cadastrado no sistema CEP/CONEP (número de aprovação 19059513.3.0000.5372 e parecer 434.935).

Tabela 1. Conteúdos da disciplina de Biologia Celular.

Apresentação do plano de ensino e Introdução ao Programa de Nivelamento.

Mundo microscópico e classificação das células.

Água e sais minerais,

Bases macromoleculares (proteínas, enzimas e carboidratos).

Bases macromoleculares (Ácidos nucléicos e lipídios).

Mitocôndrias e a produção energética celular.

Membranas celulares - estrutura e funções e Transportes através das membranas.

Lisossomos e a digestão intracelular.

Proteínas e o citoesqueleto.

Endomembranas celulares: estrutura e funções.

Núcleo celular durante o período interfásico.

Divisão celular por mitose.

Divisão celular por meiose.

Replicação do material genético.

Transcrição da informação genética.

Métodos de estudo em biologia molecular.

Células procariontes e Vírus.

Célula vegetal e Apoptose.

\subsection{PROCEDIMENTOS DOS GRUPOS CONTROLE E TRATAMENTO}

Após oito semanas de aula, a professora da disciplina aplicou uma primeira prova individual que estimava o nível de conhecimento dos estudantes sobre "biologia celular", sobre lipídios, carboidratos, organelas celulares. Os resultados desta avaliação foram divulgados no encontro seguinte, uma semana depois. Após os 
estudantes terem feito a primeira prova escrita, a professora da disciplina esclareceu a eles o experimento que pretendia fazer e todos concordaram em participar do mesmo. Foi aplicado um questionário antropométrico aos estudantes participantes do experimento no início da pesquisa, após a concordância dos mesmos em participar do projeto e da assinatura do termo de consentimento livre e esclarecido. Foi aplicado então, um teste bioquímico nos estudantes. Todos os estudantes que participaram ativamente do experimento, foram orientados a permanecerem por 12 horas de jejum. Eles tiveram uma primeira amostra de sangue capilar colhida pela manhã e em 12 horas de jejum, no início da aula e por alguns alunos da própria turma treinados, juntamente com a pesquisadora responsável pelo projeto.

Os estudantes que participaram do experimento tiveram outras duas amostras de sangue capilar colhidas 30 e 120 minutos após uma das seguintes condições: jejum (grupo jejum); ingestão de solução de glicose $1 \mathrm{~g} / \mathrm{kg}$ de peso corporal (grupo glicose); e ingestão de solução de frutose $1 \mathrm{~g} / \mathrm{kg}$ de peso corporal (grupo frutose). As soluções de frutose e de glicose foram preparadas em $5 \mathrm{ml} / \mathrm{kg}$ de peso corporal (em $5 \mathrm{ml}$ de água). $\mathrm{O}$ grupo controle apenas observou as coletas e o experimento, ou seja, vivenciou tudo que os demais estudantes fizeram, exceto não doaram sangue ou ingeriram soluções. Todos os estudantes, além de participarem ou somente observarem o experimento, estudaram os conteúdos da disciplina biologia celular através da mesma metodologia de ensino à qual os estudantes vinham se submetendo: aulas expositivas dadas pela professora, com recurso audiovisual, sendo permitida e estimulada a participação dos estudantes com perguntas e observações. Os subtemas desenvolvidos foram: lipídios, carboidratos, proteínas, enzimas e metabolismo celular, todos correlacionados ao tema central do trabalho.

Após a parte prática/experimental, todos os estudantes responderam a um questionário em sala de aula, com perguntas sobre a motivação e a importância da aula prática no aprendizado.

\subsection{AVALIAÇÃO DO DESEMPENHO E DA MOTIVAÇÃO}

Após a realização do experimento e da aplicação do questionário em sala de aula, todos os estudantes fizeram uma segunda prova individual com os conteúdos da 
disciplina biologia celular, relacionados com o experimento. As questões das provas tiveram o mesmo grau de dificuldade nas duas avaliações aplicadas aos estudantes.

\subsection{DESCRIÇÃO E ANÁLISE ESTATÍSTICA}

Para verificar a homogeneidade das variáveis qualitativas entre os grupos "Controle/Observadores" e "Ativos", foi utilizado o Teste Exato de Fisher. Para comparar as notas dos grupos antes e após a intervenção, foi utilizado o Teste de MannWhitney. Para comparar se a alteração das notas antes e depois da intervenção foi diferente entre cada um dos grupos, foi utilizado o teste de Wilcoxon. O software utilizado para a análise foi o R program versão 3.0.1.

\section{RESULTADOS}

\subsection{INDICADORES DE APRENDIZAGEM}

A aplicação de duas avaliações com o mesmo grau de dificuldade, sobre os temas da disciplina de Biologia Celular teve o objetivo analisar o impacto dos experimentos usando o próprio organismo em condições que mimetizam situações observadas no cotidiano - jejum, ingestão de refeição rica em diferentes carboidratos no rendimento nas avaliações de estudantes universitários da área da saúde.

$\mathrm{Na}$ prova que antecedeu o experimento testado neste estudo, as notas dos estudantes que participaram ativamente e dos que apenas observaram foram similares (valor-p > 0,05). Na prova realizada após o experimento, ambos os grupos tiveram aumento da nota $(\mathrm{p}<0,01)$, sendo que os indivíduos que participaram ativamente apresentaram uma nota maior do que os que apenas observaram $(\mathrm{p}<0,05$; Figura 1 e Tabela 2). 


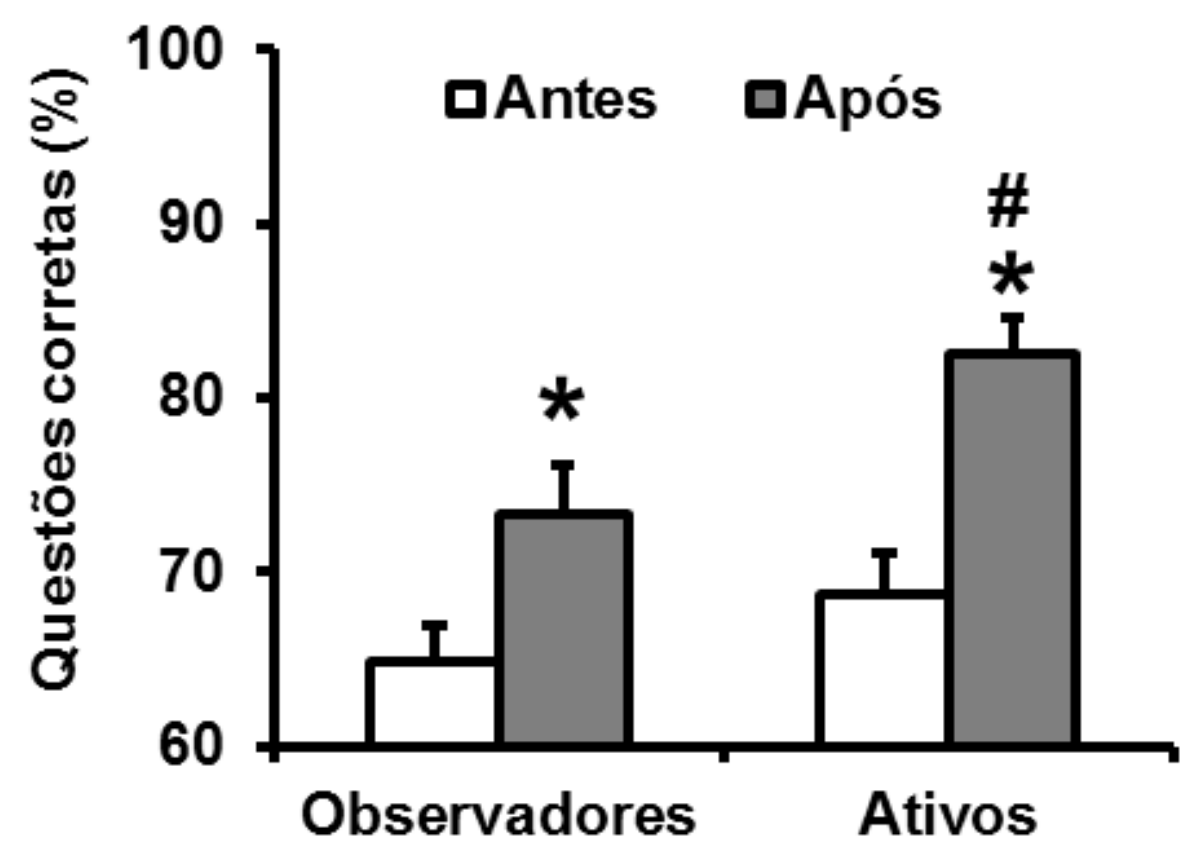

Figura 1. Efeito do experimento usando o próprio organismo de estudantes que somente observaram ou que doaram sangue sobre o seu rendimento em avaliações de aprendizagem. *: p $<0,05$. \#: $\mathrm{p}<0,05$.

Tabela 2. Medidas Descritivas para as provas A1 e A2 entre os observadores e ativos.

\begin{tabular}{ccccccccc}
\hline Variável & Part. Pesquisa & $\mathbf{N}$ & Média & E.P. & $\mathbf{1}^{\mathbf{0}} \mathbf{Q}$ & $\mathbf{2}^{\mathbf{0}} \mathbf{Q}$ & $\mathbf{3}^{\mathbf{0}} \mathbf{Q}$ & Valor-p $^{\mathbf{1}}$ \\
\hline \multirow{2}{*}{ Prova A1 } & Não & 38 & 6,49 & 0,18 & 5,50 & 6,50 & 7,00 & \multirow{2}{*}{0,074} \\
& Sim & 33 & 6,92 & 0,20 & 6,50 & 7,00 & 8,00 & \\
\hline \multirow{2}{*}{ Prova A2 } & Não & 38 & 7,33 & 0,24 & 7,00 & 7,00 & 8,00 & \multirow{2}{*}{0,017} \\
& Sim & 33 & 8,11 & 0,23 & 7,00 & 8,50 & 9,00 & \multirow{2}{*}{} \\
\hline \hline
\end{tabular}

${ }^{1}$ Teste de Mann-Whitney

Dentre os estudantes que participaram doando o seu sangue nos experimentos, não houve diferença de notas entre os três subgrupos, o que permaneceram em jejum, que ingeriram glicose e os que ingeriram frutose $(\mathrm{p}>0,05)$. Porém, somente os grupos que ingeriram glicose e frutose apresentaram aumento da nota ( $<<0,05$; Figura 2$)$. 


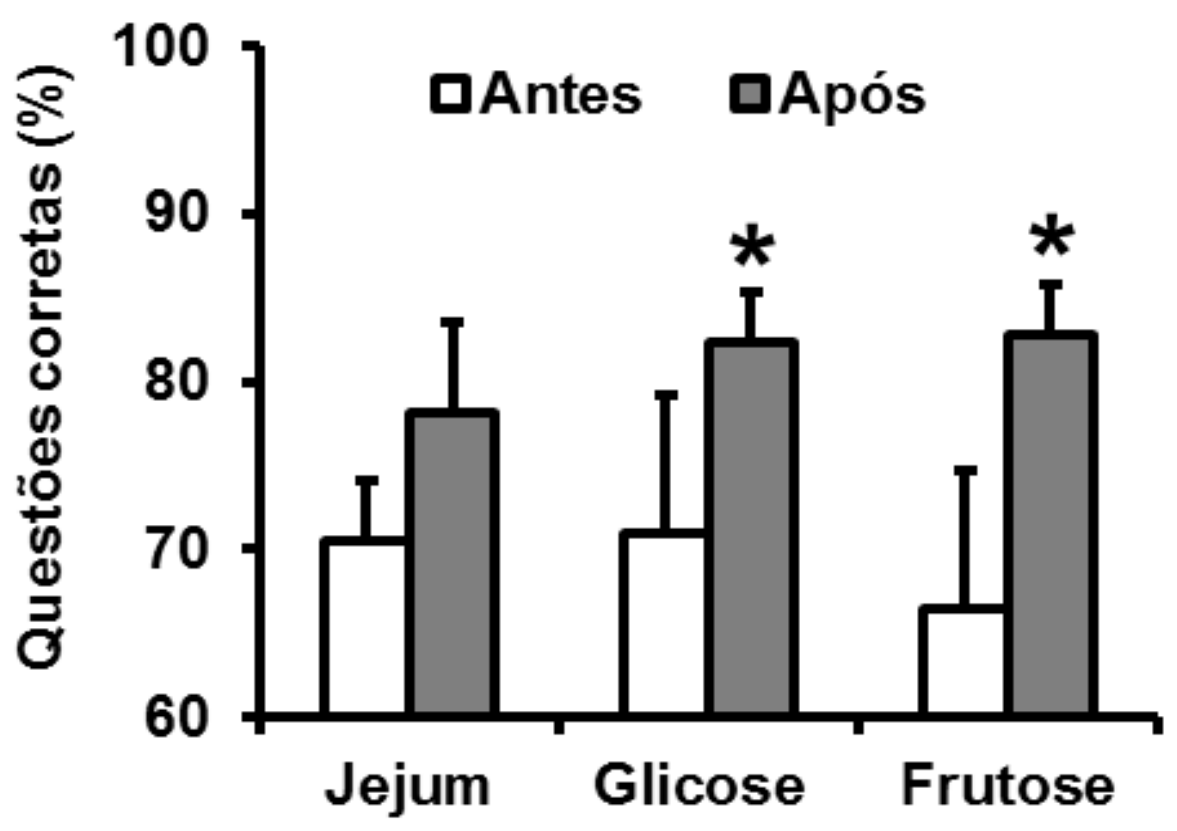

Figura 2. Efeito do experimento usando o próprio organismo de todos os estudantes que participaram ativamente doando sangue e que permaneceram em jejum ou ingeriram glicose ou frutose. $*$ : $\mathrm{p}<0,05$.

\subsection{PERCEPÇÃO SOBRE AS AULAS PRÁTICAS}

Tabela 3. Frequência e medidas descritivas para as variáveis dos ativos.

\begin{tabular}{llll}
\hline Variáveis & & $\mathrm{N}=33 \%$ \\
\hline Sexo & Feminino & 27 & $81,8 \%$ \\
& Masculino & 6 & $18,2 \%$ \\
\hline \multirow{2}{*}{ Motivação } & Baixa & 1 & $3,0 \%$ \\
& Média & 8 & $24,2 \%$ \\
\hline O aprendizado é maior quando se tem aulas & Alta & 24 & $72,7 \%$ \\
práticas & Não & 2 & $6,1 \%$ \\
\hline Aulas práticas são mais interessantes que as & Sim & 31 & $93,9 \%$ \\
teóricas & Não & 3 & $9,1 \%$ \\
\hline As aulas práticas são indispensáveis para o & Sim & 30 & $90,9 \%$ \\
aprendizado do aluno & Não & 1 & $3,0 \%$ \\
\hline & Sim & 32 & $97,0 \%$ \\
Glicose - valores das dosagens sanguíneas & Jejum & 86,0 & {$[84,5 ; 93,0]$} \\
& 30 minutos & 141,0 & {$[120,0 ;$} \\
& & & $146,0]$
\end{tabular}




\begin{tabular}{llll}
\hline & 120 minutos & 103,0 & {$[99,5 ; 119,5]$} \\
\hline Frutose - valores das dosagens sanguíneas & Jejum & 87,0 & {$[69,0 ; 90,0]$} \\
& 30 minutos & 96,0 & {$[91,0 ; 100,0]$} \\
& 120 minutos & 88,0 & {$[84,5 ; 95,0]$} \\
\hline Jejum - valores das dosagens sanguíneas & Jejum & 89,0 & {$[82,0 ; 92,5]$} \\
& 30 minutos & 98,0 & {$[90,5 ; 100,0]$} \\
\hline Idade & 120 minutos & 88,0 & {$[85,0 ; 95,0]$} \\
\hline Estatura & & 26,0 & {$[20,0 ; 33,0]$} \\
\hline Motivação em participar das aulas práticas & & 1,6 & {$[1,6 ; 1,7]$} \\
\hline \hline
\end{tabular}

Variáveis Categóricas (N, \%), Variáveis Quantitativas (Mediana, Intervalo Interquatílico)

Mais de $90 \%$ dos estudantes responderam que o aprendizado é maior quando se tem aulas práticas, que estas são mais importantes que as teóricas e que são indispensáveis. 72,7\% dos estudantes responderam ter alto nível de motivação. Porém, não houve diferença na distribuição de respostas ao questionário entre os indivíduos que participaram ativamente e os que apenas observaram (Tabela 4).

Tabela 4. Medidas Descritivas para as questões (Q1-Q5) entre os ativos e observadores.

\begin{tabular}{|c|c|c|c|c|c|c|}
\hline \multirow[b]{2}{*}{ Variáveis } & & \multicolumn{4}{|c|}{ Participou da Pesquisa } & \multirow[b]{2}{*}{$\begin{array}{l}\text { Valor } \\
\mathbf{p}^{1}\end{array}$} \\
\hline & & \multicolumn{2}{|c|}{$\begin{array}{c}\text { Não } \\
(\mathrm{N}=38) \\
\end{array}$} & \multicolumn{2}{|c|}{$\operatorname{Sim}(N=33)$} & \\
\hline \multirow{3}{*}{ Motivação } & Baixa & 4 & $10,5 \%$ & 1 & $3,0 \%$ & \multirow{3}{*}{0,427} \\
\hline & Média & 11 & $28,9 \%$ & 8 & $24,2 \%$ & \\
\hline & Alta & 23 & $60,5 \%$ & 24 & $72,7 \%$ & \\
\hline \multirow{2}{*}{$\begin{array}{l}\text { O aprendizado é maior quando se tem aulas } \\
\text { práticas }\end{array}$} & Não & 1 & $2,6 \%$ & 2 & $6,1 \%$ & \multirow{2}{*}{0,594} \\
\hline & Sim & 37 & $97,4 \%$ & 31 & $93,9 \%$ & \\
\hline \multirow{2}{*}{$\begin{array}{l}\text { Aulas práticas são mais interessantes que as } \\
\text { teóricas }\end{array}$} & Não & 5 & $13,2 \%$ & 3 & $9,1 \%$ & \multirow{2}{*}{0,716} \\
\hline & Sim & 33 & $86,8 \%$ & 30 & $90,9 \%$ & \\
\hline \multirow{2}{*}{$\begin{array}{l}\text { As aulas nas quais o aluno participa de maneira } \\
\text { ativa são mais motivadoras que as tradicionais }\end{array}$} & Não & 2 & $5,3 \%$ & 0 & $0,0 \%$ & \multirow{2}{*}{0,495} \\
\hline & Sim & 36 & $94,7 \%$ & 33 & $100,0 \%$ & \\
\hline \multirow{2}{*}{$\begin{array}{l}\text { As aulas práticas são indispensáveis para o } \\
\text { aprendizado do aluno }\end{array}$} & Não & 3 & $7,9 \%$ & 1 & $3,0 \%$ & \multirow{2}{*}{0,618} \\
\hline & Sim & 35 & $92,1 \%$ & 32 & $97,0 \%$ & \\
\hline
\end{tabular}

${ }^{1}$ Teste Exato de Fisher 


\subsection{COMPARAÇÃO DA ESCALA DE MOTIVAÇÃO}

Dentre os estudantes que participaram doando seu sangue nos experimentos, não houve diferença de motivação entre os três subgrupos, os que permaneceram em jejum, que ingeriram glicose e os que ingeriram frutose (valor-p > 0,05; Figura 4).

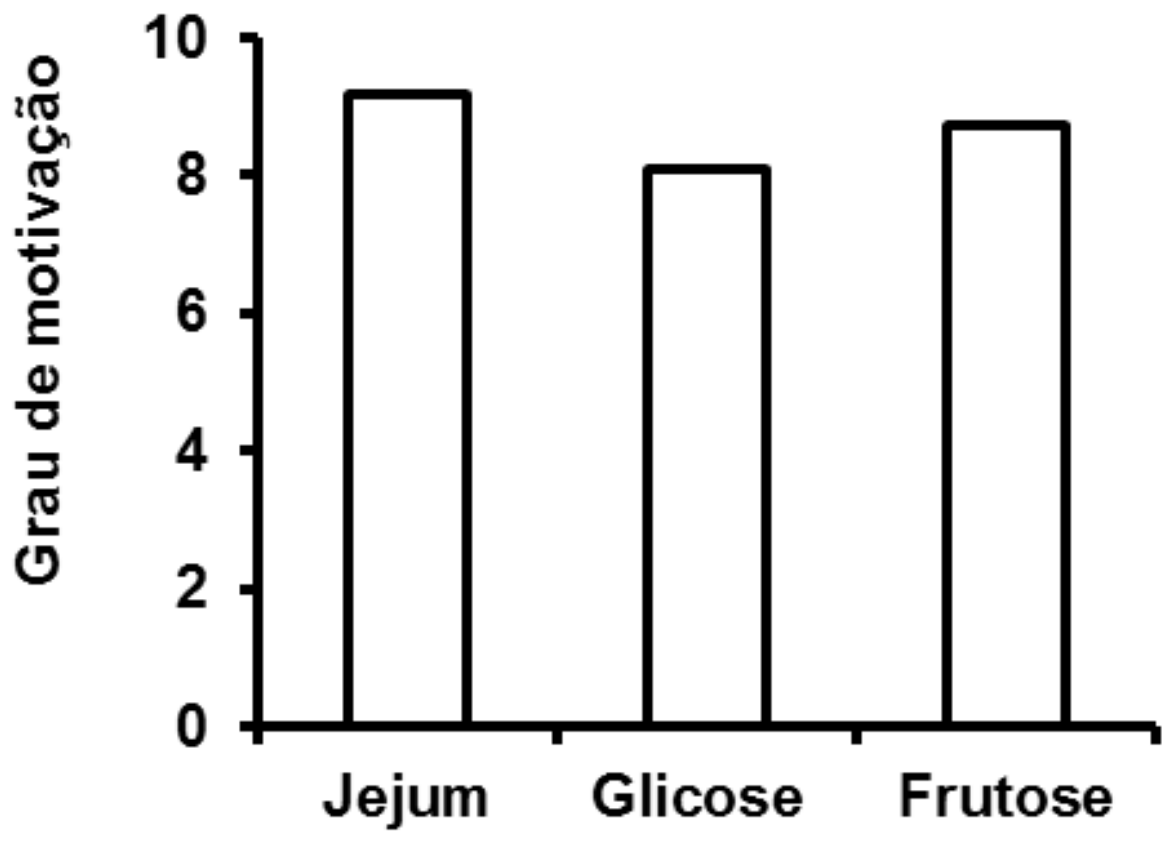

Figura 3. Grau de motivação dos estudantes que participaram ativamente doando sangue.

\section{DISCUSSÃO}

No referido estudo, analisamos o impacto de experimentos usando o próprio organismo em aulas práticas sobre o rendimento dos estudantes nas avaliações de aprendizado. Após a aplicação desse método de ensino, os grupos de estudantes que participaram ativamente das aulas práticas (usando o próprio corpo com a doação de sangue) apresentaram notas mais altas do que aqueles que apenas presenciaram. Porém, ambos os grupos, tanto o que doou sangue para as análises quanto o que apenas observou, obtiveram aumento das notas na avaliação de aprendizado. Esse aumento observado de aprendizado corrobora muitos autores que advogam a favor dos métodos de aprendizagem ativa e vários trabalhos que mostraram um aumento da aprendizagem com seu uso $(12,33,9,23)$. Porém, a maioria dos artigos nos quais os autores afirmam que, metodologias de aprendizagem ativa são melhores do que outras, se baseia em teorias, pois não mostra resultados de experimentos envolvendo ensino ou não utilizou 
grupos controle adequados (24). Vários estudos com adequado grupo controle não observaram diferenças de aprendizado através de metodologias de ensino com aprendizagem ativa, em comparação aos métodos tradicionais mais passivos $(16,29,7)$. Há estudos que mostram que o uso de alguns métodos ativos de aprendizagem pode resultar em notas até mais baixas $(6,30)$. Essas diferenças de resultados obtidos com os diferentes métodos de aprendizagem indicam que há outros aspectos que interferem sobre a sua eficiência e que podem ocorrer independentemente do método de ensino ser mais ou menos ativo. Um deles parece ser a conduta dos professores. Por exemplo, uma melhoria do ensino tende a ocorrer quando os professores colocam problemas e formulam perguntas, e estimulam um clima de trabalho que permita aos alunos liberdade para arriscar, errar e aprender com os próprios erros (5). De fato, a atratividade das atividades didáticas desenvolvidas é maior com a presença física do professor (32, 2). Desta forma, é esperado que as estratégias de aprendizagem que proporcionem as melhores contingências de aprendizado sejam mais eficientes. $O$ resultado mais marcante do presente estudo foi o maior aumento de notas do grupo de estudantes que participou ativamente das aulas práticas em relação àquele que apenas presenciou.Vale ressaltar que todos os grupos estavam juntos durante todo o experimento. Esse achado condiz com os resultados positivos observados de métodos que envolvem a auto avaliação do aprendizado pelos próprios estudantes (1). Na verdade, até onde sabemos, este é o primeiro estudo que mostra que um método de ensino no qual o estudante avalia o próprio corpo promove um maior aprendizado. Outros estudos já haviam indicado que aulas envolvendo o próprio corpo trariam benefícios $(26,22)$, porém, ainda não havia um estudo com grupos controle. Uma explicação para este fenômeno seria que o método inclui um grande envolvimento compulsório do estudante, o que provavelmente aumenta o seu grau de atenção e foco no experimento realizado e evita dispersões/distrações.

Os resultados obtidos pelos estudantes durante a prática puderam mostrar a eles como o organismo responde a situações comuns do cotidiano. Nos grupos que ingeriram carboidratos, houve aumento da glicemia e este aumento foi maior no grupo que ingeriu glicose do que no grupo que ingeriu frutose. Assim como no nosso experimento, estudos diversos foram feitos para medir a glicemia frente a soluções de glicose e frutose $(3,4$, $35)$, e frente a alimentação do cotidiano das pessoas $(26,22)$. A ausência de diferença de notas da prova posterior ao experimento entre os três subgrupos que participaram 
ativamente (que permaneceu em jejum, que ingeriu glicose ou que ingeriu frutose) indica que a coleta do próprio sangue aumentou a compreensão e retenção das informações de toda a prática e não somente do resultado do seu próprio organismo ou do seu grupo. Desta forma, aqueles que ingeriram glicose também compreenderam o que ocorre ao se ingerir frutose ou se permanecer em jejum. Possivelmente, isso se deveu à maior atratividade juntamente com a maior atenção proporcionadas pela metodologia empregada. Outro dado que corrobora ainda mais isso é o da análise dos três subgrupos que participaram ativamente do experimento. $\mathrm{O}$ fato de não ter havido aumento significativo de nota no subgrupo que permaneceu em jejum, mas somente nos que ingeriram glicose e frutose, indica que a ingestão de soluções fez com que os estudantes tivessem um maior aprendizado do que aqueles que somente doaram sangue. A metodologia utilizada neste estudo envolve diferentes formas de participação ativa e ao mesmo tempo um estudo sobre o próprio corpo em situações vivenciadas diariamente, proporciona uma aprendizagem ativa visceral.

Surpreendentemente, o grau de motivação e satisfação em realizar experimentos, medido pelo questionário aplicado, não foi diferente entre os diferentes grupos. Este resultado era inesperado, pois métodos de aprendizagem mais ativa tendem a gerar maior interesse $(14,13)$. Porém, a ausência de diferença pode se dever ao fato de que os estudantes que não utilizaram o próprio corpo nas práticas também participaram do experimento (como expectadores). Esta explicação é corroborada pelo fato de que mais de $90 \%$ dos estudantes responderam que as aulas são mais interessantes e que o aprendizado é maior quando se tem aulas práticas. Outros estudos mostraram valores diferentes, por exemplo, estudantes e residentes de medicina e participantes da metodologia de aprendizagem ativa se mostraram mais engajados, com maior interação entre os alunos (43\%) e com o instrutor (57\%), mas relataram uma menor percepção dos objetivos da aprendizagem (16). Em outro estudo, a maioria dos estudantes de medicina da disciplina de bioquímica clínica (73\%) opinou que a metodologia ativa de aprendizagem foi motivadora, despertou o interesse na disciplina e aumentou o aprendizado (18). Outra explicação para esses resultados do nosso trabalho é uma possível insensibilidade das perguntas usadas no questionário para percepções sutis dos estudantes. Talvez, a aplicação de questões qualitativas poderia ajudar a definir melhor a relação entre motivação e o método de ensino. 
Conclui-se no presente estudo que uma metodologia ensino de aprendizagem ativa, na qual os estudantes usam o próprio organismo e sangue nas aulas práticas, é capaz de aumentar as notas dos estudantes em provas de conhecimento, indicando um aumento do aprendizado, quando comparados ao grupo controle, que apenas observou as experiências. Além disso, a análise dos três subgrupos que participaram ativamente do experimento mostrou que aqueles que ingeriram soluções de glicose e frutose tiveram aumento significativo de nota, enquanto que o que permaneceu em jejum não. Tal diferença indica que a metodologia associando duas estratégias diferentes, a aprendizagem ativa e a auto avaliação, aumenta o grau de atenção às aulas. $\mathrm{O}$ instrumento de ensino apresentado no presente trabalho mostrou ser uma alternativa positiva para estudantes universitários da área da saúde.

\section{REFERÊNCIAS BIBLIOGRÁFICAS}

1- Agrawal S, Norman GR, Kevin W E.Influences on medical students's self-regulated learning after test completion. Blackwell Pub Ltd. Med Ed.; 46: 326-35, 2012.

2- Ali WG. E. Caring and Effective Teaching Behavior of Clinical Nursing Instructors in Clinical Area as Perceived by Their Students. J Educ Prat.: 3(7) 15-27, 2012.

3- Abraha A, Sandy M. Humphreys, Mo L. Clark, David R. Matthews e Keith Frayn N.. Efeito agudo da frutose na lipemia pós-prandial em indivíduos diabéticos e nãodiabéticos. British Journal of Nutrition, 80, pp 169-175, 1998.

4- Bidwell, Amy J., Impact of Physical Activity on Postprandial Lipidemia and Glycemic Control after Chronic Fructose Ingestion. Exercise Science Dissertations. Paper 8., 2012.

5- Biggs J, and Catherine Tang. Teaching for quality learning at university: What the student does. McGraw-Hill Education (UK), 2011.

6- Brewer, D. J.,\&Goldhaber, D..Whydon't schools and teachers seem to matter? The Journal of Human Resources, 32(3), 505-523, 1997.

7- Chao SH, Brett B, Wiecha JM, Norton LE, Levine SA. Use of an online curriculum to teach delirium to fourth-yaer medical studentes: a comparison with lecture format. J Am Geriatr Soc.: 60(7):1328-32, Jul 2012. 
8- Consorti et al.: Evaluation of the acceptability of Peer Physical Examination (PPE) in medical and osteopathic students: a cross sectional survey. BMC Medical Education 13:111, 2013.

9- Cruz SCS, CarvalhoAAA. Produção de Vídeo com o Movie Maker: Um Estudo Sobre o Envolvimento dos Alunos de 9. ${ }^{\circ}$ Ano na Aprendizagem. 2007.

10- Dobson, John L., Tracy Linderholm. "The effect of selected "desirable difficulties" on the ability to recall anatomy information." Anatomical sciences education (2014).

11- Edelbring S, Broström O, Henriksson P, Vassiliou D, Spaak J, Dahlgren LO, Fors U, Zary N. Integrating virtual patients into courses: follow-up seminars and perceived benefit. Med Educ.;46(4):417-25, Apr 2012.

12- Erwin TD, Rieppi R. Comparing multimedia and traditional approaches in undergraduate psychology classes. Teaching of Psychology.; 26:58-61, 1999.

13- Everly MC. Are students' impressions of improved learning through active learning methods reflected by improved test scores?Nurse Educ Today.: 33(2):148-51, Feb 2013.

14- Ghosh S, Dawka V. Combination of didatic lecture with problem-basead learning sessions in physiology teaching in a developing medical college in Nepal. Adv Physiol Educ.: 24(1):8-12, Dec 2000.

15- Goldhaber, Dan D., and Dominic J. Brewer. "Does teacher certification matter? High school teacher certification status and student achievement. Educational evaluation and policy analysis 22.2: 129-145, 2000.

16- Haidet P, Morgan RO, O'Malley K, Moran BJ, Richards BF. A Controlled Trial of Active Versus Passive Learning Strategies in a Large Group Setting. Adv Health Sci Educ Theory Pract. ;9(1):15-27, 2004.

17- Harasym PH, Tsai TC, Munshi FM. Is problem-based learning an ideal format for developing ethical decision skills? Kaohsiung J Med Sci.;29(10):523-9, Oct 2013.

18- Joshi, K. B., Nilawar, A. N., \& Thorat, A. P. . Effect of case based learning in understanding clinical biochemistry. International Journal of Biomedical and Advance Research, 5(10), 516-518, 2014.

19- Khan BA, Ali F, Vazir N, Barolia R, Rehan S. Studentes perceptions of clinical teaching and leraning strategies: a Pakistani perspective. Nurse Educ Today. 32(1):85-90, Jan 2012. 
20- Kulak, V. e Newton, G., Um guia para usar baseado em casos de aprendizagem no ensino de bioquímica. Biochem. Mol. Biol. Educ., 42: 457-473, 2014.

21- Larsen DP, Butler AC, Roediger HL III. Repeated testing improves long-term retention relative to repeated study: a randomised controlled trial. Med Educ 2009; 43 (12):1174-81.

22- Mari K. Hopper, Luke W. Maurer. Laboratory exercise: study of digestive and regulatory processes through the exploration of fasted and postprandial blood glucose. Advances in Physiology Education 37(3): 254-263, 2013.

23- Narula N, Ahmed L, Rudkowski J. An evaluation of the '5 Minute Medicine' video podcast series compared to conventional medical resources for the internal medicine clerkship. Med Teach.; 34(11): 751-5, 2012.

24- Nascimento GPV. Estudo Controlado da Efetividade de um Instrumento que Acopla Aprendizagem Ativa e Tecnologia: Criação de Vídeos pelos Estudantes. Dissertação de Mestrado, UnB. Brasília: 2014.

25- Parikh A, McReelis K, Hodges B. Student feedback in problem based learning: a survey of 103 final year students across five Ontario medical schools. Med Educ 35: 632-636, 2001.

26- Passos RM, Sé AB, Wolff VL, Nobrega YK, Hermes-Lima M. Pizza and pasta help students learn metabolismo. Adv Physiol Educ.; 30(2):89-93, Jun 2006.

27- Prince KJAH, Van Eijs PWLJ, Boshuizen HPA, Van Der Vleuten CPM, and Scherpbier AJJA. General competencies of problem-based learning (PBL) and nonPBL graduates. Med Educ 39: 394-401, 2005.

28- Rivers, W. P. . Autonomy at all costs: An ethnography of metacognitive selfassessment and self- management among experienced language learners. The Modern Language Journal, 85(2): 279-290, 2001.

29- Schreiber BE, Fukuta J, Gordon F.Live lecture versus video podcast in undergraduate medical education: A randomised controlled trial.BMC Med Educ: 10:68, 2010.

30- Schwerdt, G., \& Wuppermann, A. C.. Is traditional teaching really all that bad? A within-student between-subject approach. Economics of Education Review, 30(2), 365-379, 2011. 
31- Stevenson FT, Bowe CM, Gandour-Edwards R, and Kumari VG. Paired basic science and clinical problem-based learning faculty teaching side by side: do students evaluate them differently? Med Educ 39: 194-201, 2005.

32- Warwick P, Mercer N, Kershner R, Staarman JK. In the mind and in the technology: the vicarious presence of the teacher in pupil's learning of science in collborative group at the interactive whiteboard. Computers \& Education 55: 350$62,2010$.

33- Wiecha JM, Chetty VK, Pollard T, Shaw PF. Web-based Versus Face-to-Face Learning of Diabetes Management: The Results of a Comparative Trial of Educational Methods. Fam Med:;38(9):647-52, Oct. 2006.

34- Yang, W. M., \& Xu, X. Z. . Self assessment in second-language learning. USChina Foreign Language: 6(56), 20-24, 2008.

35- Zawiasa A, Nowicki M, Acute effects of fructose consumption on uric acid and plasma lipids in patients with impaired renal function, Metabolism: 62(10): 14621469, Oct. 2013. 


\section{DISCUSSÃO GERAL E CONCLUSÕES}

No ensino superior, onde transformações em busca de se aprimorar o aprendizado e aumentar a participação dos estudantes ocorrem constantemente, os docentes tem papel fundamental neste processo. No entanto, a qualificação dos professores (por exemplo, graus, a experiência, certificações e o desempenho no teste de professores) mostra apenas as relações modestas para o desempenho dos alunos (Béteille, 2009; Early et al., 2005). Sendo assim, a introdução de novas tecnologias na educação deve ser seguida por análise e adaptação de práticas (Edelbring, 2010).

O principal método de ensino utilizado pelos professores nas salas de aula sempre foi a tradicional palestra didática (Lujan e DiCarlo, 2006). Os métodos passivos de aprendizagem em sua maioria não incluem interação entre os estudantes, sendo o intuito principal apenas oferecer aos estudantes uma determinada matéria do curso em questão (Wilke RR, 2003). Convém ressaltar, que a presença física do professor e a sua relação direta com os alunos é que faz com que a metodologia tradicional de ensino seja ainda a preferida em salas de aula.

Uma alternativa pertinente de promover a melhora do ensino é através do uso de metodologias ativas (Springer et al., 1997). O conhecimento sobre os estilos de aprendizagem pode ajudar os educadores a identificar e resolver os problemas de aprendizagem entre os estudantes, ajudando, assim, os seus alunos a se tornarem aprendizes mais eficazes (Collins, 2004). Qualquer estilo de aprendizagem adotado por um professor não é preferível nem inferior a outro, mas é simplesmente diferente, com diferentes pontos fortes e fracos característicos de cada um (Felder e Brent, 2005). Além disso, uma série de estudos tem detectado alterações nas estratégias de aprendizagem com o tempo (Phan, 2011; Reid et al., 2005; Vanthournout, 2011).

O aumento da aprendizagem e da motivação dos alunos em participar das aulas onde metodologias de ensino de aprendizagem ativa foram empregadas, foi comprovado em diversos estudos publicados na literatura (Rao et al., 2000; Wang et al., 2010; Obrez et al., 2011). Também no nosso experimento, chegamos a este mesmo resultado. As notas obtidas nos exames realizados após as aulas práticas foram maiores do que as que antecederam o experimento. 
Para os estudantes que participaram ativamente e para os que apenas observaram, houve um aumento significativo na pontuação da prova pós-experimento, se comparado com a prova realizada antes do experimento, sendo que pelo menos $50 \%$ dos que participaram ativamente tiveram um aumento menor ou igual a 1,5 pontos na prova pós-experimento quando comparado com a nota da prova realizada antes do experimento. Enquanto que dos indivíduos que somente observaram, pelo menos $50 \%$ tiveram um aumento menor ou igual a 1,0 ponto na prova posterior à prática quando comparado a nota da prova realizada antes da aplicação da prática. Em relação ao questionário aplicado aos estudantes, sobre a motivação e a satisfação em realizar experimentos, não houve evidências de diferença significativa na distribuição de respostas das questões (Q1-Q5) entre os que participaram ou não da pesquisa. Embora os valores medianos para os grupos glicose e frutose, tenha sido maiores que do grupo jejum, não houve diferença significativa das notas de ambas as provas entre os grupos.

Fica claro que obstáculos ainda devem ser superados para a implantação de aulas com metodologias de aprendizagem ativa, sejam elas em laboratório ou em sala de aula. O professor tem papel fundamental na participação dos alunos e na motivação da turma, de uma maneira geral. Tal como acontece com qualquer intervenção educacional, as diferentes metodologias de aprendizagem devem ser entendidas não isoladamente, mas em relação ao envolvente contexto educacional. A diversidade de práticas metodológicas é que faz com que o ensino esteja em constante aperfeiçoamento. Cabe aos discentes e às instituições de ensino, sejam elas privadas ou públicas, buscarem sempre e de maneira incessante, métodos que tornem o aprendizado do aluno mais eficiente e prazeroso, seja ele da maneira tradicional ou através de uma nova metodologia baseada na aprendizagem ativa. 


\section{RECOMENDAÇÕES PARA ESTUDOS FUTUROS E CONCLUSÃO}

Apesar de diversos estudos relacionados à utilização de metodologias ativas terem sido publicados nos últimos anos, estudos que mostrem resultados concretos de experimentos envolvendo ensino ou que utilizem grupos controle adequados,

permanecem ainda pouco explorados. É sabido que o ensino superior é um momento único na vida de todo estudante, pois se trata de uma fase cheia de inseguranças, e em muito isto se deve a expectativa criada em torno da futura vida profissional. Os membros do corpo docente devem apresentar interesse em melhorar seus conhecimentos e aprender mais sobre o uso da aprendizagem ativa na sala de aula, com o intuito principal de aumentar o aproveitamento do aluno no ensino superior. Esta pesquisa teve como objetivo geral analisar o impacto de experimentos usando o próprio organismo em condições que mimetizam situações observadas no cotidiano - jejum, ingestão de refeição rica em diferentes carboidratos - sobre o rendimento nas avaliações e no grau de motivação em realizar estes experimentos. É importante destacar que este estudo limitou-se a 71 alunos do curso de farmácia de uma instituição privada de ensino superior de Brasília e os resultados obtidos fazem referência à realidade. A metodologia de ensino de aprendizagem ativa pode trazer resultados positivos e significativos quando bem utilizada. Apesar dos resultados encontrados terem se mostrado significativamente positivos quanto às notas obtidas pelos alunos que participaram da aula prática de maneira ativa ou apenas como observadores, tais resultados não devem ser extrapolados ou generalizados para quaisquer populações.

O fato da grande maioria dos estudantes, participantes ou não da parte prática do exeperimento, ter relatado através do questionário aplicado que as aulas são mais interessantes e o aprendizado é maior através de experiências práticas, apoia a teoria de que a metodologia de aprendizagem ativa pode ser mais benéfica do que métodos mais passivos de ensino.

Sugere-se, então, que mais pesquisas sejam realizadas nesta área com o intuito de colaborar para o aperfeiçoamento das técnicas de ensino, como por exemplo: estudos semelhantes a este poderiam ser realizados, comparando-se alunos de um determinado curso em instituições de ensino superior privado e público. Ou comparar o aprendizado 
de estudantes que não vivenciaram experimentos com outros que vivenciaram e que participaram com diferentes graus de envolvimento (como no presente estudo no qual alguns apenas doaram sangue enquanto que outros ingeriram carboidratos).

Conclui-se com esse estudo que, frente aos resultados positivos encontrados através da aplicação de uma metodologia de ensino de aprendizagem ativa, com estudantes universitáros do curso de farmácia de uma instituição privada de ensino de Brasília, independente dos mesmos participarem ativamente ou apenas como observadores, este novo instrumento de ensino pode ser considerado como eficiente. Tal metodologia pode ser aprimorada e aperfeiçoada, com o objetivo de contribuir ainda mais para o aumento do aprendizado dos discentes. 


\section{REFERÊNCIAS BIBLIOGRÁFICAS (das partes não pertencentes ao manuscrito)}

Barker SP. Comparison of effectiveness of interactive videodisco versus lecture demonstration instruction.Phys Ther. May,1988; 68(5):699-703.

Boyer, R. The new biochemistry: blending the traditional with the other, Biochem. Mol. Biol. Educ.2000; 28, 292-296.

Collins J. Education techniques for lifelong learning: principles of adult learning. Radiographics. 2004; 24:1483-1489.

D. Early et al., Pre-Kindergarten in Eleven States: NCEDL's Multi-State Study of Pre-Kindergarten and State-Wide Early Educational Programs (SWEEP) Study National Institute for Early Education Research, Washington, D. C., 2005.

Felder R, Brent R. Understanding student differences. Journal of Engineering Education.2005; 94(1): 57-72.

Feuerwerker LC. Além do discurso de mudança na educação médica: processos e resultados. São Paulo: Hucitec; 2002.

Figueira AC, Rocha JB. A proposal for teaching undergraduate chemistry students carbohydrate biochemistry by problem-based learning activities. Biochem Mol Educ., 2013.

Hermes-Lima M, Muniz KC, and Coutinho IS. The relevance of student seminars on clinically related subjects in a biochemistry course for medical and nutrition students. Biochem Mol Biol Educ. 2002; 30: 30-34.

Lujan HL, DiCarlo SE. First-year medical students prefer multiple learning styles. Adv Physiol Educ. 2006; 30: 13-16.

Obrez A, Briggs C, Buckman J, Goldstein L, Lamb C, Knight WG. Teaching clinically relevant Dental Anatomy in the Dental Curriculum: description and assessment of an innovative module. J. Dent. Educ. Jun 2011; 75(6):797-804.

Persky AM, Pollack GM. A modified team-based learning phisiology course. Am J Pharm Educ. Dec 2011; 75(10):204. 
Phan HP, Deep processing strategies and critical thinking: Developmental trajectories using latent growth analyses. The Journal of Educational Research. 2011; 104: 283-294.

Rao, S.P. and DiCArlo, S.E. Peer Instruction Improves Performance on Quizzes. Advances in Physiology Education. 2000; 24: 51-55.

Reid WA, Duvall E, Evans P. Can we influence medical students' approaches to learning? Medical Teacher. 2005; 27: 401-407.

Rivkin A, Gim S.Student preferences regarding teaching methods in a drug-induced diseases and clinical toxicology course.Am J Pharm Educ. Aug 2013; 77(6): 123.

Rozendaal JS, Minnaert A, Boekaerts M. Motivation and self-regulated learning in secondary vocational education: Information-processing type and gender differences. Learning and Individual Differences. 2003; 13: 273-289.

Saville B, Zinn T, Neef N, Van Norman R, Ferreri S. A comparison of inter-teaching and lecture in the college classroom. Journal of Applied Behavior Analysis.2006; 39:49-61.

Springer L, Stanne ME, Donavan S. Measuring the success of small-group learning in college-level SMET teaching: a meta-analysis. National Institute for Science Education. 1997.

T. Béteille, S. Loeb, in Handbook of Education Policy Research, G. Sykes, B. Schneider, D. N. Plank, Eds. Routledge, New York. 2009; 596-612.

van Bragt CAC, Bakx AWEA, Van der Sanden JMM, Croon ME. Students' approaches to learning when entering higher education: Differences between students with senior general secondary and senior secondary educational backgrounds. Learning and Individual Differences. 2007; 17: 83-96.

Vanthournout G. Patterns in student learning: Exploring a person-oriented and longitudinal research-perspective. Antwerp: Garant. 2011; 220 p.

Wang J, Zhang W, Qin L, Zhao J, Zhang S, Gu J, Zhou C. Problem-Based Learning in Regional Anatomy Education at Peking University. Anat Sci Educ. May- Jun 2010; 3(3):121-6.

Wilke RR. The effect of active learning on student characteristics in a human physiology course for nonmajors. Adv Physiol Educ. 2003; 27: 207-223. 


\title{
12. ANEXOS
}

ANEXO A - APROVAÇÃO DO COMITÊ DE ÉTICA EM PESQUISA

\section{ANHANGUERA EDUCACIONAL Plotoformo $S A / S P$}

\section{PARECER CONSUBSTANCIADO DO CEP}

\section{DADOS DO PROJETO DE PESQUISA}

Título da Pesquisa: Análise da resposta do próprio corpo a um experimento metabólico e o seu efeito sobre o aprendizado

Pesquisador: Anna Maly de Leão e Neves Eduardo

Área Temática:

Versão: 2

CAAE: 19059513.3 .0000 .5372

Instituição Proponente: ANHANGUERA EDUCACIONAL LTDA

Patrocinador Principal: Financiamento Próprio

\section{DADOS DO PARECER}

Número do Parecer: 434.935

Data da Relatoria: 21/10/2013

\section{Apresentação do Projeto:}

Trata-se da V.2 do protocolo de pesquisa postado a fim de atender as seguintes recomendações do CEP

1. Proceder à correção ou explicar a Frase: "Evidência de relação direta entre os resíduos sólidos urbanos e a saúde dos catadores de materiais recicláveis do Distrito Federal" que se encontra na brochura do

\begin{abstract}
3. Rever o título da pesquisa: "A influência das aulas práticas no aprendizado dos estudantes universitários da área da saúde" que parece estar dissociado dos objetivos: - Analisar o impacto dos experimentos usando o próprio organismo em condições que mimetizam situações observadas no cotidiano - jejum, ingestão de refeição rica em lipídeos e/ou carboidratos no rendimento nas avaliações dos estudantes universitários da área da saúde. Avaliar o grau de motivação e satisfação em realizar experimentos investigando a resposta do organismo ao jejum e
\end{abstract}

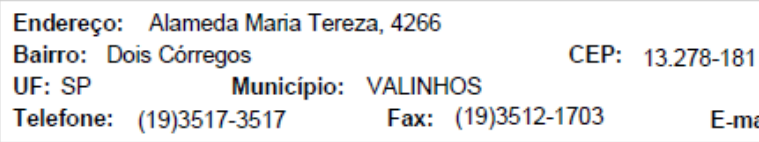


à ingestão de diferentes alimentos.

4. TCLE: Postar novo TCLE que descreva com mais detalhes sobre a coleta de sangue (volume/ via), bem como garantir no termo o uso de descartáveis e que o procedimento será realizado conforme previsto nas normas de segurança de saúde.

O atendimento às recomendações encontra-se nos campos pertinentes à suas análises.

\section{Objetivo da Pesquisa:}

Não cabe análise do item na versão em questão, pois não haviam pendências a ele relacionadas.

\section{Avaliação dos Riscos e Benefícios:}

Não cabe análise do item na versão em questão, pois não haviam pendências a ele relacionadas.

Comentários e Considerações sobre a Pesquisa:

Foi retirada a frase ¿Evidência de relação direta entre os resíduos sólidos urbanos e a saúde dos catadores de materiais recicláveis do Distrito Federal" que se encontra na brochura do pesquisador (em resultados esperados) e no Protocolo de Pesquisa (desfecho primário);

Foi retirada a citação "Normas de Biossegurança da UnB". Ficou claro que a pesquisa será realizada apenas na Faculdade Anhanguera de Brasílica. Desta forma não houve necessidade de autorização de coparticipante.

O título da pesquisa foi modificado para: Análise da resposta do próprio corpo a um experimento metabólico e o seu efeito sobre o aprendizado

\section{Consideraçōes sobre os Termos de apresentaçäo obrigatória:}

TCLE:

Foi postado novo TCLE que descrevendo detalhes sobre a coleta de sangue (volume/ via), e garantindo o uso de descartáveis e que o procedimento será realizado conforme previsto nas normas de segurança de saúde.

\section{Recomendações:}

O projeto está aprovado nos moldes apresentados. Eventuais modificações ou emendas ao protocolo devem ser apresentadas ao CEP de forma clara e sucinta, identificando a parte do protocolo a ser modificada e suas justificativas.

Conclusões ou Pendências e Lista de Inadequaçōes:

O Comitê de Ética em Pesquisa da Anhanguera Educacional, após acatar o parecer do membro- 
reıator previamente aesıgnaao para o presente projeto, veriıcar o atenaımento de toaos os aısposituvos aas Resoluções 466/2012 e complementares, aprovar o Termo do Consentimento Livre e Esclarecido e todos os anexos incluídos na Pesquisa, resolve aprovar sem restrições o Protocolo de Pesquisa supracitado.O conteúdo e as conclusões aqui apresentados são de responsabilidade exclusiva do CEP/AESA e não representam a opinião da Anhanguera Educacional nem a comprometem.

Situação do Parecer:

Aprovado

Necessita Apreciação da CONEP:

Não

Considerações Finais a critério do CEP:

O sujeito da pesquisa tem a liberdade de recusar-se a participar ou de retirar seu consentimento em qualquer fase da pesquisa, sem penalização alguma e sem prejuízo ao seu cuidado e deve receber uma cópia do Termo de Consentimento Livre e Esclarecido, na integra, por ele assinado. O pesquisador deve desenvolver a pesquisa conforme delineada no protocolo aprovado e descontinuar o estudo somente após análise das razões da descontinuidade pelo CEP que o aprovou, exceto quando perceber risco ou dano não previsto ao sujeito participante. O CEP deve ser informado de todos os efeitos adversos ou fatos relevantes que alterem o curso normal do estudo. É papel do pesquisador assegurar medidas imediatas adequadas frente a evento adverso grave ocorrido (mesmo que tenha sido em outro centro) e enviar notificação ao CEP e à Agência Nacional de Vigilância Sanitária ¿ ANVISA (quando couber) junto com seu posicionamento. Eventuais modificações ou emendas ao protocolo devem ser apresentadas ao CEP de forma clara e sucinta, identificando a parte do protocolo a ser modificada e suas justificativas. Em caso de projeto do Grupo I ou II apresentados anteriormente à ANVISA, o pesquisador ou patrocinador deve enviar as modificações para esse órgão junto com o parecer aprovatório do CEP, para serem juntadas ao protocolo inicial. Relatórios parciais e final devem ser apresentados ao CEP, de acordo as Resoluções vigentes.

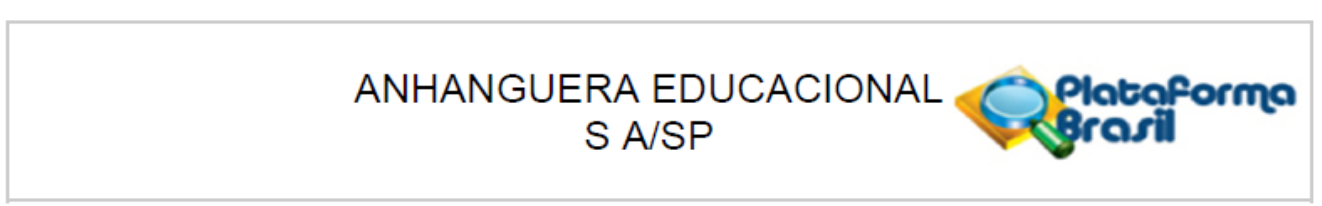

Continuação do Parecer. 434.935

VALINHOS, 24 de Outubro de 2013

Assinador por:

Mariane Bernadete Compri Nardy

(Coordenador) 


\section{ANEXO B - TERMO DE CONSENTIMENTO LIVRE E ESCLARECIDO (TCLE)}

\section{Termo de Consentimento Livre e Esclarecido $\quad \mathbf{N}^{\mathbf{o}}$}

Análise da resposta do próprio corpo a um experimento metabólico e o seu efeito sobre o aprendizado

Você está sendo convidado para participar como voluntário de um projeto de pesquisa. I - O presente estudo tem como objetivo analisar o impacto dos experimentos usando o próprio organismo em condições que mimetizam situações observadas no cotidiano jejum, ingestão de refeição rica em lipídeos e/ou carboidratos no rendimento nas avaliações dos estudantes universitários da área da saúde. Avaliar o grau de motivação e satisfação em realizar experimentos investigando a resposta do organismo ao jejum e à ingestão de diferentes alimentos. E será realizado pela professora e pesquisadora Anna Maly de Leão e Neves Eduardo.

II - Sua participação na pesquisa será voluntária, sem qualquer tipo de gasto, e consistirá em responder perguntas de um questionário referentes ao seu conhecimento sobre metabolismo, importância das aulas práticas, e hábitos alimentares. Além disso, será coletada uma amostra de sangue para realização de exames bioquímicos. Todos os materiais utilizados serão descartáveis e estéreis, para garantir a segurança do processo. Vale ressaltar que seu nome será mantido em total sigilo e que você gastará cerca de 10 a 20 minutos para o preenchimento do questionário.

III - Durante a pesquisa, você poderá elucidar suas dúvidas relacionadas ao questionário, assim como poderá desistir de participar da pesquisa em qualquer momento desejado. Caso queira obter informações sobre os resultados da pesquisa e esclarecer dúvidas sobre a importância do estudo, entre em contato com a pesquisadora do projeto Prof. Anna Maly de Leão e Neves Eduardo.

IV - Os dados e resultados obtidos com as respostas do questionário e mediante os procedimentos realizados na pesquisa poderão ser publicados, mas seus dados pessoais serão mantidos em sigilo. 


\section{Caso necessite entre em contado com o CEP Anhanguera Educacional:}

End: Alameda Maria Tereza, 2000 - Dois Córregos. Valinhos - S.P.

CEP: 13.278-181. Fone: (19) 3512-4815. Fax: (19) 3512-1703

e-mail: cep@unianhanguera.edu.br

$\mathrm{Eu}$, após ter

recebido informações sobre o estudo: A influência das aulas práticas no aprendizado dos estudantes universitários da área da saúde, por meio da carta informativa lida por mim ou por terceiro, declaro que ficaram claros os objetivos do estudo, os procedimentos a serem realizados, as garantias de confidencialidade e de esclarecimentos permanentes. Não tendo dúvidas a respeito da pesquisa, concordo tomar parte como voluntário no estudo, do qual posso deixar de participar a qualquer momento sem penalidades ou prejuízos, ou perda de qualquer benefício que possa ter adquirido.

Este termo de consentimento será assinado em duas vias, sendo a primeira de posse do participante da pesquisa e a segunda dos pesquisadores.

Assinatura do participante

Data: do pesquisador

Data: Assinatura 


\section{ANEXO C - TERMO DE RESPONSABILIDADE E COMPROMISSO DA PESQUISADORA RESPONSÁVEL}

Eu, Anna Maly de Leão e Neves Eduardo, pesquisadora responsável pelo projeto: Efeito de experimentos em aulas práticas com o corpo dos próprios estudantes sobre o seu nível de aprendizagem, declaro estar ciente e que cumprirei os termos da Resolução 196, de 09/10/96, do Conselho Nacional de Saúde do Ministério da Saúde, e declaro: (a) assumir o compromisso de zelar pela privacidade e sigilo das informações; (b) tornar os resultados desta pesquisa públicos, sejam eles favoráveis ou não; e, (c) comunicar o CEP sobre qualquer alteração no projeto de pesquisa, nos relatórios anuais ou através de comunicação protocolada, que me forem solicitadas.

Brasília, 30 de março de 2013.

Anna Maly de Leão e Neves Eduardo 
ANEXO D - QUESTIONÁRIO

\section{QUESTIONÁRIO:}

NOME:

PARTICIPOU ATIVAMENTE DA PRÁTICA DE BIOCEL EM JEJUM?: （ ） SIM ( ) NÃO

1- Motivação em participar de aulas práticas utilizando o próprio organismo? ( )alta ( ) média ( ) baixa nota de 0 a 10 :

2- Em sua opinião, o aprendizado é maior quando se tem aulas práticas na disciplina?

( ) $\operatorname{sim}$ ( ) não

3- As aulas práticas são mais interessantes que as aulas teóricas?

( ) $\operatorname{sim}$ ( ) não

4- As aulas nas quais o aluno participa de maneira ativa são mais motivadoras que as tradicionais?

( ) $\operatorname{sim}$ ( ) não

5- As aulas práticas são indispensáveis para o aprendizado do aluno?

( ) $\operatorname{sim}$ ( ) não

COMENTÀRIO: 
ANEXO E - CLASSIFICAÇÃO QUALIS CAPES DA REVISTA “ADVANCES IN PHYSIOLOGY EDUCATION"

\begin{tabular}{|c|l|c|l|l|}
\hline ISSN & \multicolumn{1}{|c|}{ TÍTULO } & ESTRATO & $\begin{array}{r}\text { ÁREA DE } \\
\text { AVALIAÇÃO }\end{array}$ & STATUS \\
\hline $1043-4046$ & $\begin{array}{l}\text { Advances in Physiology } \\
\text { Education }\end{array}$ & A1 & ENSINO & Atualizado \\
\hline
\end{tabular}




\section{ANEXO F - INSTRUÇÕES A AUTORES PARA SUBMISSÃO DE MANUSCRITO À REVISTA “ADVANCES IN PHYSIOLOGY EDUCATION”}

\section{Manuscript submission}

Instructions to Authors: Physiology Sourcebook 1 Advances in Physiology Education LABORATORY SOURCEBOOK Instructions to Authors Please read carefully as some of these instructions differ from the standard APS guidelines. A Word template based on these instructions can be downloaded from the Advances website to facilitate writing and submission. Explanations of the different elements in a Sourcebook activity are in italics. • The headers in BOLD must be part of the manuscript. • Use of the underlined subheaders in the manuscript is optional but all information indicated by the subheaders should be included if relevant. - Text should be double-spaced and pages numbered sequentially. - Figures and supplemental information should be uploaded in separate files. Indicate in the body of the manuscript the insertion place for each figure or table, TITLE (160 characters and spaces maximum) FIRST AUTHOR, ADDITIONAL AUTHORS Department and Institution, City and State or Country affiliations for each author. (If author affiliations have changed since the work was done, include current affiliations in the Acknowledgments section of the submission.) CONTACT INFORMATION FOR CORRESPONDING AUTHOR (full mailing address, phone, fax, and email information for the author who will take responsibility for the submission) ABBREVIATED TITLE for the running head (60 characters and spaces maximum) KEYWORDS (3-5 words or short phrases that do not appear in the title or running head) ABSTRACT (250 WORD LIMIT). INTRODUCTION: A descriptive review of the physiology underlying the activity Objectives and overview: A brief paragraph introducing the activity and what occurs during the activity. Background: The Background should contain all information needed to educate the reader about the activity and it may need to be quite detailed. What phenomena does the activity explore? What physiological parameters are measured or observed? Relate these phenomena to physiological principles. If possible, include citations for the original research from which the activity derives. Do not simply cite previously published versions of the activity.Instructions to Authors: Physiology Sourcebook 2 Place the activity and its 
physiology in the broader context, with particular attention to factors that might interest students. Think of how you might answer a student who asks "Why are we doing this activity?" The Background may include figures, graphs, and/or flow charts created by the author(s), or those for which the authors have obtained permission to reprint. Users of Sourcebook activities should not have to consult outside references to understand the relevant physiology as they may not have access to appropriate resources. Because the Sourcebook targets high school teachers and instructors who teach introductory biology in addition to physiologists, you should assume that the reader has minimal background in physiology when explaining the background. Where possible, select references that are freely available through the internet, such as publications more than a year old in the American Physiological Society journals. Learning Objectives: A numbered list that includes both content knowledge objectives and process skills. After completing this lesson, the student will be able to: [CONTENT KNOWLEDGE: Describe the key concepts students will learn from the activity. Objectives should begin with action verbs such as explain / describe / list / draw, etc.] [PROCESS SKILLS: [Describe key skills to be learned from activity. Objectives should begin with action verbs such as measure / design / predict / analyze, etc.] Activity Level: What level of student or what classes or courses might use this activity? Prerequisite Student Knowledge or Skills: Students should have a basic understanding of: [list of background content students should know to successfully complete this exercise] Students should know how to: [list of skills or techniques students should have mastered prior to beginning this activity] Time Required: How long will this activity take? Can it be done in one session or does it require several sessions? METHODS: Equipment and Supplies This section should contain all the information needed to set up and run the activity. Assume the reader has no experience in this subject and needs to set up the activity for the first time. For example, a local teacher calls you and asks for the instructions but has never done this type of activity before and cannot come see you for a demonstration. What would this teacher need to Instructions to Authors: Physiology Sourcebook 3 know to buy the equipment and supplies, assemble them, make solutions, and run the activity successfully? - Directions should be detailed and should take the form of a numbered, annotated list. • Descriptions must be specific and should include acceptable ranges of dimensions. For example, "rubber tubing" is not specific. A better description is " $3-5$ ' of 
$1 \mathrm{~cm}$ I.D. rubber or plastic tubing." - Provide alternatives when possible. For example: "handheld or computer-linked sphygmomanometer." (Adopters in some teaching situations may not have access to computers or specialized equipment.) - Include manufacturers and model numbers for equipment. - Include o where to purchase unusual items o instructions for building simple equipment o lists of chemical needed and source if unusual o recipes for solutions and concentrations of solutions $o$ Photographs or illustrations showing equipment and how it is arranged or used. This section may include original diagrams and photographs or those used with permission. Photographs with identifiable people over the age of 18 require permission to use from the subjects. - For animal activities, please include any information needed on their purchase, care while being held for the activity and appropriate disposal of the animals at the conclusion of the activity. Human and Animal Subjects • If the activity uses humans or vertebrate animals, please include a statement about protocol approval or exemption by the author's institutional review board or animal care and use committee. All activities using vertebrate animals or students as human subjects must include the following statement: Adopters of an activity are responsible for obtaining permission for human or animal research from their home institution. For a summary of Guiding Principles for Research Involving Animals and Human Beings, please see www.theaps.org/mm/Publications/Ethical-Policies/Animal-and-Human-Research Appropriate anesthesia or analgesia should be described for all animal activities, including those using invertebrates. Experiments using students as human subjects should be noninvasive or minimally invasive. Instructions to Authors: Physiology Sourcebook 4 Instructions This section must provide enough detail so that the process can be duplicated easily. - It should be written in the form of a numbered list of steps that may include annotations. Numbered steps allow you to reference a particular step later in the discussion. - This section should also include samples of data collection forms or report forms if appropriate. - This section should include diagrams or photographs if appropriate. Troubleshooting Use this section to describe common student mistakes and what can go wrong. Explain how to correct or prevent technical problems. List sources of possible artifacts or erroneous results. Safety Considerations: Please indicate if students should be excluded as experimental subjects. If animals are used, are there any precautions needed in handling them? If chemicals are used, include 
a summary of the critical Material Safety Data Sheet (MSDS) information for each one. What precautions are needed in handling or disposing of chemicals? List protective gear required (gloves, goggles, etc.). For example: Diazoxide has the following MSDS warnings: may be harmful if swallowed, causes skin irritation, causes serious eye irritation, may cause respiratory irritation. Students should wear appropriate personal protection equipment, including gloves, goggles, and mask if handling the dry powder. RESULTS This section is critical for adopters who have never done this activity before. Expected Results: This should begin with an explanation of what you would expect students to observe or measure. Relate the results to the physiology. Provide sample data or graphs if possible. Explain what the results will look like when students make common mistakes. If sample data sets for an entire class are available, these could be useful as preparatory work or for a 'dry lab' activity. Please consider including these data sets as supplemental information (see later section on SUPPLEMENTAL MATERIAL.) Misconceptions: Any there any misconceptions that might be corrected by doing this activity? Evaluation of Student Work This section should include • how students might present their dataInstructions to Authors: Physiology Sourcebook 5 • examples of questions to be answered o If answers to questions are not found elsewhere in this submission (for example, in the Introduction), they should be included here, in brackets immediately after the question. • prompts for a lab report or other written work Inquiry Applications What is the inquiry level of the lesson? (see list below) Consider the following points: - Who decides the question to be explored? - Who plans the procedure to be used? Sometimes this is a combination of a basic protocol provided by the teacher and variations on that protocol developed by the students. $\bullet$ Who carries out the experiment and analyzes the data? "Methods" level: Teacher generates the question(s) and teacher designs the experiment. "Facilitated Inquiry": Teacher provides guidelines for the research questions and experimental design. Students expand on the question and methods. "Guided inquiry": Teacher generates the question(s) and students design the experiment. Students may modify the teacher-generated question. "Open inquiry": Students generate the question and students design the experiment. How can the activity be made more student-centered and/or used at a higher inquiry level? Please describe some examples of how this could be done. Can the lesson serve as an introduction to a method that can then be used in higher level inquiry investigations? 
Provide suggestions for follow-up activities when appropriate. Wider Applications: Can the activity be used in a problem-based learning context or case-based learning context? Provide detailed examples when appropriate. Additional Information: This section may include a description of how to handle the class discussion once the activity is done. It may also include additional interesting background information about the activity or about the physiology behind the activity. This section can also be used to relate the physiology to real-world applications, such as medical uses for the techniques. ADDITIONAL RESOURCES: Should include original source citations, textbooks, and such that have not been previously cited in the submission. • Add additional resources to your REFERENCES list and add their reference list numbers to the following statement:Instructions to Authors: Physiology Sourcebook 6 For additional information on this topic, please see (reference list citation numbers for the items). Resources should be easily accessible so that adopters without extensive library resources can find them. Search the APS journals website (www.the-aps.org/mm/Publications/Journals) for reviews and other related articles. - As with any paper that cites the work of others, please do not include references that you have not personally read. • Please limit the use of web sites whose URLs might not be stable. - Papers that are published in print journals but accessed online should have the print volume citation, not the access URL. Search the APS Archive of Teaching Resources at www.apsarchive.org for related items, such as other labs, Advances in Physiology Education articles, Legacy articles, etc. Add these to the REFERENCES list and add their reference list numbers to the following statement: For additional resources related to this experiment, please see (reference list citation numbers for the items). ACKNOWLEDGMENTS: Use this section to acknowledge others who have been involved in the development of this activity. Thank people who have given permission for use of figures. Include current author address information here if it has changed from title page address. REFERENCES This section follows the standard citation format for APS journals. Reference lists should be arranged alphabetically by author and numbered serially. The reference number should be placed in parentheses at the appropriate place in the text. The examples given below are for the most common source types. For a complete list of examples, see www.the-aps.org/mm/Publications/Preparing-YourManuscript\#references Journal article: Author AB. Title of article. Journal Title 
Abbreviated vol: pp-pp, year. Book: Author AA, Author B, and Author CC. Title (x ed.). City: Publisher, date. Chapter in a book: Author AA and Author CC. Title of the chapter. In: Book Title, edited by Editor AA, Editor BB. City: Publisher, date, p. 0000.Instructions to Authors: Physiology Sourcebook $7 \mathrm{Web}$ article: Author/editor (if known). (Revision or copyright date, if available). Title of page [Publication medium]. Page publisher. URL (Protocol://Site/Path/File) [Access date]. Access date may be general (year only) or to the day. If a reference is available both in print and online in electronic format, always cite the print reference. FIGURES: Figures should be submitted as pdf files. For complete and detailed instructions, including guidelines and rules for the use of animals and humans in photographs, please see http://www.theaps.org/mm/Publications/Preparing-Your-Manuscript/Preparing-Figures

SUPPLEMENTAL MATERIAL Video and audio files, long data sets, and similarly cumbersome material that cannot be feasibly published in a PDF page may be submitted for inclusion in the online journal (without charge to the author) as supplemental material. Such material must be submitted for peer review along with the manuscript and must meet the approval of the journal Editor. For all supplemental materials, authors should include a caption for each file, explaining the purpose and content of the file. Electronic publication of the Sourcebook activities allows authors the flexibility to include video and audio files or large sample data sets that could be used by students to analyze results if they do not have the equipment needed to carry out the activity. Material that can be set into an article in standard APS house style, such as figures, tables, equations, and other material that can be easily copyedited and typeset into our final-published PDF page, may NOT be submitted as supplemental data. Such material must be incorporated into the article as standard figures or tables. Audio/Video: Authors are responsible for compiling their own digital audio or video. Each file should be no more than 10 megs in size. Authors may be required to resubmit video files with shorter running time, smaller frame size, or lower resolution in order to conform to the recommended file size. Long Data Sets: Long data sets should be submitted in Microsoft Excel or in Microsoft Word table format. Authors should include a title and legend explaining the content and purpose of each data set. 